\title{
Does Personality effect Facial Emotion Recognition? A Comparison between the older Ekman Emotion Hexagon Test and a newly created Measure
}

\author{
Laura Jenkins* \\ Northumbria University, Department of Psychology, Newcastle-upon-Tyne, UK
}

\section{Article Info}

\author{
*Corresponding author: \\ Laura Jenkins \\ Northumbria University \\ Department of Psychology \\ Newcastle-upon-Tyne \\ UK \\ E-mail: laurajenkins840@gmail.com
}

Received: October 24, 2017

Accepted: November 10, 2017

Published: November 16, 2017

Citation: Jenkins L. Does Personality effect Facial Emotion Recognition? A Comparison between the older Ekman Emotion Hexagon Test and a newly created Measure. Madridge J Neurosci. 2017; 1(1): 38-46.

doi: $10.18689 /$ mjns -1000107

\section{Copyright: (c) 2017 The Author(s). This work is licensed under a Creative Commons Attribution 4.0 International License, which permits unrestricted use, distribution, and reproduction in any medium, provided the original work is properly cited.}

Published by Madridge Publishers

\begin{abstract}
Recent research has highlighted one possible problem faced when it comes to assessing the recognition of emotion in the human face. Previous research has suggested that the images used in the methods of assessment are becoming too familiar within the psychological research domain. They therefore suggest new ways of creating images to look at facial emotion recognition.

To investigate this issue, the current study created a new emotion recognition task (Laura's Emotion Hexagon Task) and compared this to the older Ekman's Emotion Hexagon Test. 60 participants completed both tasks along with the IPIP-NEO. This measure looked at whether personality could predict the scores for the two facial emotion recognition tasks. Results showed that the agreeableness and extraversion personality were significant predictors of Laura's Emotion Hexagon Task. Discussions of the findings are in relation to previous research about methodological issues surrounding facial emotion recognition, and results are discussed in relation to previous findings of how personality can effect facial emotion recognition.
\end{abstract}

Keywords: Facial emotion; Human face; Laura's Emotion Hexagon; Ekman's Emotion Test.

\section{Introduction}

Emotion recognition has been researched for many years, dating back to the late $1800 \mathrm{~s}$. One of the first theorists to conduct research about the expression of emotion was Darwin [6]. Darwin wrote a book describing how emotions can be in voluntary in all animals and humans. He focused more upon the biological reasons for displays of emotion, relating it to both animal and human species. This was the first real topic for literature critiques to look at within the field of emotion recognition.

In recent years, Cacioppo and Gardener [3] have given their accounts of emotion and looked at the issue with a more psychological perspective. The study looked at how human emotion is measured. They reviewed the past literature that had aimed to discover more about human emotions from different perspectives. Cacioppo and Gardener [3] described different methodologies of measuring emotion, such as fMRI techniques. A review of the literature, which looked at how situational variables influence emotion. One perspective involved looking at literature that investigated emotion using PET scans [12]. Researchers also looked at how intelligence links in some way to emotion recognition [15].

When discussing emotion recognition, it is very important to look at the research conducted by theorists such as Ekman. Ekman, Friesen and Tomkins [11] had also looked at past research relating to Darwin [6]. They noted that Darwin had mainly focused on the 
biological reasons of how emotional expressions can occur in the human face (for example, which muscles and tissues are in use during expression). Conclusions suggest that research should have a slight change in direction. Ekman, Friesen and Tomkins decided to investigate the emotions that observers could see in the human face (for example, happiness or disgust) instead of focusing on how the emotions themselves are displayed and what muscles are used. This gave several opportunities to question how researchers could actually measure emotion recognition in the human face, therefore inspiring Ekman to create several measures and methodologies to help measure emotion recognition in faces.

One emotion recognition test is named as Ekman's 60 Faces Test. In this test, participants have to decide which emotion is being displayed in a series of photographs. The photographs are taken from 'Pictures of Facial Affect' [7]. Ekman's 60 Faces Test was created with 10 participants (models) who had six different facial expression photographs taken. The expressions were happiness, anger, sadness, fear, disgust and surprise, and Ekman had identified all as the six basic human emotions in his earlier work. As the name of the test suggests, Ekman's 60 Faces Test contains 60 facial photographs so therefore participants can obtain a total score of 60 . A score of 60 would mean that a participant had an excellent ability to recognise the emotions in human faces.

Other researchers investigated the uses of Ekman's 60 Faces Test by looking to see whether the test could be used as a diagnostic tool to highlight any problems with facial emotion recognition in patients who had frontotemporal dementia [7]. Results demonstrated that Ekman's 60 Faces Test discriminates between patients with frontotemporal dementia and healthy patients when it came to recognise emotions in the human face.

The research conducted by Ekman [9] was not the only line of investigation that was taking place. Matsumoto, LeRoux, Wilson-Cohn, Raroque, Kooken, Ekman, Yrizzary, Loewinger, Uchida, Yee, Amo and Goh [17] had attempted to create a new way of measuring facial emotion recognition. They designed the Japanese and Caucasian Brief Affect Recognition Test (JACBART) to help researchers understand and measure people's individual differences in emotion recognition ability. Matsumoto had worked with Ekman in previous years to create measures such as the Japanese and Caucasian Facial Expressions of Emotion (JACFEE) and Neutral Faces (JACNeuF). Literature surrounding these measures had shown differing results lacking in validity and reliability. Matsumoto and colleagues therefore decided to create a new measure (JACBART) and included improvements to the criticism made earlier in their research. They used photographs that had good validity and reliability, and included photographs of people who were of a variety of ethnic origins.

One notable criticism of facial emotion recognition methods was that the same series of photographs were being used on multiple occasions. The photographs were taken from 'Pictures of Facial Affect', which was created by Ekman and Friesen [11].
Research (Suzuki, Hoshino and Shigemasu) [20] had suggested that the non-morphed images used form 'Pictures of Facial Affect' [11] were becoming too easy to recognise. They therefore proposed a morphing technique which could increase the difficultly level of the emotion recognition photographs.

Ekman's Emotion Hexagon Test has been created so that individuals would have to choose one emotion out of a photograph that would have contained two different emotions. The photographs, used in 'Pictures of Facial Affect' were morphed to form images that contained two emotions out of the six basic emotions (happiness, sadness, anger, fear, surprise and disgust). It was suggested that an individual would find Ekman's Emotion Hexagon Task more challenging than his 60 Faces Task because the different features that could be recognised in each emotion were less apparent.

Ekman's Emotion Hexagon Test was shown that it could be used successfully [4]. Researchers were investigating human facial emotion recognition across the adult life span so therefore created two mini studies to do this. Study 1 used the original photos created for Ekman's 60 Faces Task. Study 2 used the morphed images created for Ekman's Emotion Hexagon Test instead of the non-morphed images as described above. Results showed that with increasing age, participants found it more difficult to identify five out of the six basic emotions. The emotion of disgust was the opposite of this. With increasing age, participants found it easier to interpret the emotion of disgust in faces.

Ekman's Emotion Hexagon Test has very good reliability and validity. The FEEST Manual [22] gives details of both Ekman's 60 Faces Task and more importantly for this study; it gives details about Ekman's Emotion Hexagon Test.

Split-half reliability scores demonstrated reliability. 40 participants were shown the images used in the Emotion Hexagon Test to ensure that each emotion could be successfully recognised. Only 50 percent of the images were reliable in allowing a participant to identify emotions in the human face. Only this 50 percent of photographs were used in Ekman's Emotion Hexagon Test and the photographs that were unsuccessful were taken out of the procedure. Reliability techniques (split-half reliabilities) gave statistical significance for the emotion of sadness, anger, fear, disgust, and surprise.

The validity of Ekman's Emotion Hexagon Test is successful due to the use of it on many occasions. Many empirical studies [4] [2] [11], have managed to use the series of images to create Ekman's Emotion Hexagon Test. This gives the test a very good rate of validity as each individual study used the pictures to show the correct displays of emotion to different participants.

Research has also looked at Ekman's Emotion Hexagon Test [5]. This investigation looked at strategies used when participants have to choose a certain emotion in the human face. They concluded that it was a very advantageous to include some forced-choice answers. For example, in Ekman's Emotion Hexagon Test, participants have to choose from a variety of six basic emotions instead of just writing down the 
ne displayed emotion. Researchers noted that this was a very good way of assessing emotion recognition in faces as it eliminates the choice of simply guessing the answer.

With the issue of universality of previous photographs becoming more apparent, new measures help researchers to identify new relationships within the field of emotion recognition in faces. This is, therefore, one of the main aims of the current study, to create a new and updated measure of emotion recognition.

The present researchers had noted that for a successful comparison to take place there had to be a specific variable to measure. In recent years, these variables have included aspects such as emotional intelligence, personality and age.

Austin [2] used pictures taken from Ekman and Friesen $[10,11]$ to show that emotional intelligence correlated with emotion facial recognition. Researchers had used Ekman's 60 Faces Task and the Trait Emotion Intelligence Questionnaire to show these relationships.

Terracciano, Merritt, Zonderman and Evans [21] had demonstrated that the personality trait of openness can also affect emotion facial recognition and concluded that the more open an individual is, then the easier they can successfully identify the basic emotions in the human face. Researchers aimed to replicate a past study conducted by Matsuomo et al. [17] who had conducted research using Caucasian and Asian students. Terracciano et al. [21] used the NEO Five Factor Inventory to assess each individual's personality traits, and used two sub-tasks of the Perception of Affect Task (PAT). The two sub tasks included images, whichwas taken from the research of Ekman and Friesen. Results did demonstrate that openness could affect an individual's facial emotion recognition and that conscientiousness had a similar (but smaller) effect.

Mill, Allik, Realo and Valk [18] looked at how openness could affect an individual's facial emotion recognition. They were initially looking at age related differences in emotion recognition but came across the finding that both openness and conscientiousness could influence facial emotion recognition. They used the measures Japanese and Caucasian Facial Expressions of Emotion (JACFEE) and Neutral Faces (JACNeuF), created by Matsumoto and Ekman [16], which included the pictures used in Ekman's work. Researchers also used the NEO-FFI [1] which assessed the Big Five Factors of Personality in each participant. Both openness and conscientiousness were found to have positive correlations with emotion recognition. This indicates that the more open and conscientious a person is then the better they will do on the facial emotion recognition tasks.

\section{Aims and Predictions}

The overall aim of this investigation is to create a new and modern version of Ekman's Emotion Hexagon Test and name this Laura's Emotion Hexagon Task. Laura's Emotion Hexagon Task will have the same layout as Ekman's Emotion Hexagon Test, using one male volunteer who agreed to have facial emotive photographs taken. The male volunteer had six facial emotion photographs taken, similar to Ekman's - expression of happiness, sadness, anger, fear, disgust and surprise.

Due to the most recent literature focussing on how personality can relate to facial emotion recognition, the variable of personality is going to be used to help compare Ekman and Laura's tasks. To do this, researchers will use the IPIP-NEO (Goldberg) [13] to measure the Big Five Factors of Personality. Goldberg et al. [14] discussed the development of the IPIP-NEO (Goldberg) [13]. The IPIP-NEO allows people in the public domain to have access to a personality test that was simple to administer. This is one of the reasons as to why the current investigation uses the IPIP-NEO. No extensive administer training is needed to administer the test so therefore it is a simple but effective way of assessing an individual's personality type. The variable of personality will be measured because this area of psychology also has an increasing number of research studies to support the relationship between personality and emotion facial recognition. Examples include research such as Terracciano et al. [21].

From this study, it is predicted that there will be a positive relationship between openness and both Laura's task score and Ekman's task score. The more open an individual is then the higher their scores will be on both Ekman's Emotion Hexagon Test and Laura's Emotion Hexagon Task. It is also predictions that openness will be a predictor of both Laura's and Ekman's task scores. A final hypothesis is that Laura's Emotion Hexagon Task will be significantly correlated with Ekman's Emotion Hexagon Test.

\section{Method}

\section{Design and Rationale}

A $5 \times 2$ repeated measures design is used for the purposes of this experiment. There are two factors for the experiment. The first factor is 'personality' and this has five levels being 'openness', 'conscientiousness', 'extraversion', 'agreeableness' and 'neuroticism'. The level of 'openness' will be split into two categories of 'low openness' and 'high openness'. This is so that researchers can have a more detailed look at whether facial emotion recognition is predicted by a person's openness. The second factor is 'task type' and this has two levels. The first level is 'Laura's Emotion Hexagon Task' and the second level is 'Ekman's Emotion Hexagon Test'. There is only one dependent variable in the experiment and this is the 'score of the tasks'. The scores of each task will be noted separately (and not added together) so that a comparison can take place.

The second factor of 'task type' will use a repeated measures design as participants are required to complete both Laura's Emotion Hexagon Task and Ekman's Emotion Hexagon Task. This will allow a comparison to between both tasks. A between subjects' design is being used on the first factor of 'openness score'. This is because participants can only fall into one of the two categories of 'high openness' or 'low openness'. 


\section{Participants}

Before investigations could take places, two participants (one male and one female volunteer) had their photographs taken for the creation of Laura's Emotion Hexagon Task. These two participants did not take part in any other phase of the investigation. Researchers decided not to use the photographs taken from the female volunteer as Ekman's Test only used males.

Sixty experimental participants (5 males and 55 females) were recruited from a North East University so that testing could begin. Participants had to be students of the university and ideally aged between 18 and 65 years of age.

\section{Materials}

The IPIP NEO [13] was used to assess participants' personality types. This comprised of a 120 item multiplechoice questionnaire that was given to participants as an online version.

Participants were given Ekman's Emotion Hexagon Task (2002) to assess their emotion recognition abilities within facial photographs. This was a computer-based task, given to participants by the use of a CD-ROM. The task comprised of 150 facial photographs of which participants had to decide which basic emotion was present. The photographs consisted of six different facial emotion expressions that had been morphed to create a series of new images (photographs) for a male model. Each new photograph (morphed) contained a total of two basic emotions (ranging from 10-90 percent ratios between the displays of emotions). Participants had to note down which emotion was present. Participants' maximum score was 120 (20 points from each emotion expression). Images displaying expressions with a 50:50 emotion ratio were not counted in the points score. Participants had one practice trial consisting of 30 photographs (points were not counted at this stage); then had five test trials (points were counted at this stage).

To create Laura's Emotion Hexagon Task, photographs were taken of a male volunteer expressing the five basic emotions of happiness, sadness, anger, surprise and disgust. As this task was looking at morphed facial images, the software Phanta Morph was used to combine the emotions in the facial images. Each emotion facial photograph contained two facial expressions that could be identified and there were a total of sixty photographs created (thirty for the male participant and thirty for the female participant). After the computer programme had been created, researchers decided to omit the photographs taken from the female volunteer so that a more appropriate comparison could take place.

Laura's Emotion Hexagon Task (2010) was given to participants to assess their emotion recognition abilities within facial photographs. Please see figure 1 for an example of the male emotive faces. This task was a computer based programme consisting of 150 photographs from one male volunteer. Each photograph contained two emotion expressions which had been morphed into one photograph.
There were six possibilities of emotion expression - happiness, sadness, anger, fear, surprise and disgust. The scoring was the same as Ekman's Emotion Hexagon Task, where participants would not be scored for a photograph that had been created with an emotion expression ratio of 50:50. The total score for Laura's Emotion Hexagon Task was 120 (20 points maximum coming from each one of the six basic emotion expressions).

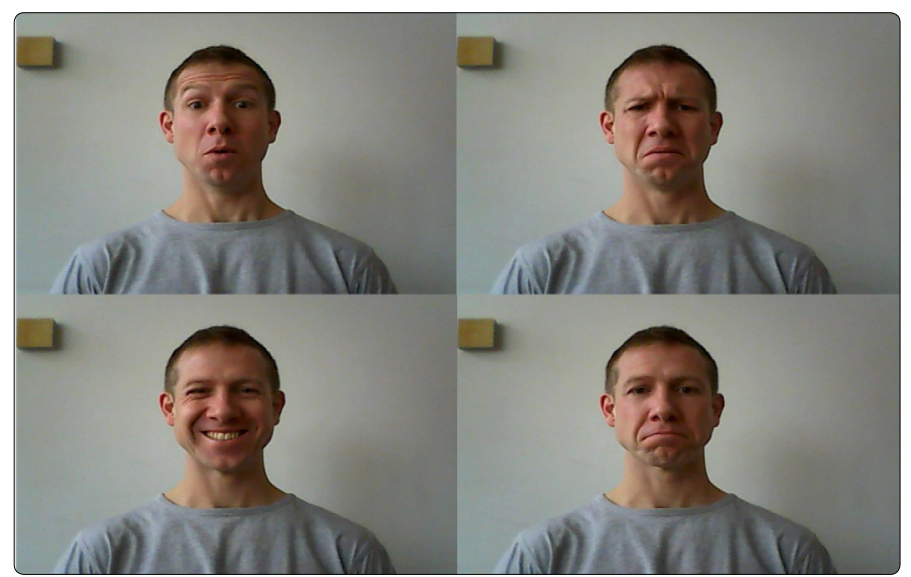

Figure 1. Example of the images used in Laura's Emotion Hexagon Task.

Bottom right is the expression of sadness, bottom left is the expression of happiness. Top left is the emotion of surprise and top right is the emotion of disgust

\section{Procedure}

Participants were contacted to arrange an appropriate meeting time and place for testing. This was normally in the seating area of the $3^{\text {rd }}$ Floor Northumberland Building at Northumbria University as it was a quiet area used for independent learning.

Participants were asked to read the participant information sheet so that they could discover the aims behind the investigation. Participants were informed that they could withdraw from the investigation at any point up until the data was sent for analysis.

When participants were satisfied, they signed a consent form so that researchers had a clarification that participants were willing to take part.

When this was completed, testing could begin. Participants completed the IPIP-NEO (Goldberg) [13]. This was an online multiple-choice questionnaire, which assessed each participant's personality.

Participants completed Ekman's Emotion Hexagon Task (2002). Please see list of materials for explanation of the task. This task comprised of a practice phase (where scores were not noted) and then five testing phases where scored were noted. This took approximately ten minutes.

Participants completed Laura's Emotion Hexagon Task (2010). This was the second emotion recognition task using 150 morphed photographs of facial expressions. Please see materials for explanation of the task. This again took approximately 10 minutes and the practice trials did not receive any score. 
After all testing had been completed, participants were given a full debrief using the participant debrief sheet. This was a two page debrief which detailed the full aims of the investigation and gave a chance for participants to ask questions. Once again, participants were reminded that they could withdraw from the investigation within the allocated time limit (before results were analysed).

If participants had ticked the appropriate box on the consent form, they were then informed that they would be notified of the overall results of the investigation, and that they could contact the researchers at any point with queries.

\section{Results}

Sixty participants provided data from completing both Laura's Emotion Hexagon Task and Ekman's Emotion Test. Each test could have a maximum score of 120 . Participants' personality trait scores of openness, conscientiousness, agreeableness, extraversion and neuroticism were also collected. Each personality trait could have a maximum score of 100 .

\section{Evaluation of Overall Task}

Table 1. Mean scores (and Standard Deviations) of all variables, including personality traits and both emotion hexagon tests.

\begin{tabular}{|c|c|c|c|c|c|c|}
\hline $\begin{array}{c}\text { Laura's } \\
\text { Task }\end{array}$ & $\begin{array}{c}\text { Ekman's } \\
\text { Test }\end{array}$ & $\begin{array}{c}\text { Open- } \\
\text { ness }\end{array}$ & $\begin{array}{c}\text { Conscientious- } \\
\text { ness }\end{array}$ & $\begin{array}{c}\text { Extraver- } \\
\text { sion }\end{array}$ & $\begin{array}{c}\text { Agreeable- } \\
\text { ness }\end{array}$ & $\begin{array}{c}\text { Neuroti- } \\
\text { cism }\end{array}$ \\
\hline $81(12.1)$ & $99(19.2)$ & $\begin{array}{c}21 \\
(19.0)\end{array}$ & $\begin{array}{c}46 \\
(24.9)\end{array}$ & $\begin{array}{c}50 \\
(25.3)\end{array}$ & $\begin{array}{c}55 \\
(26.0)\end{array}$ & $\begin{array}{c}47 \\
(24.4)\end{array}$ \\
\hline
\end{tabular}

\section{Table Notes}

The first section of the analysis was a correlation between Laura's Emotion Hexagon Test and Ekman's Emotion Hexagon Test. This correlation showed a positive (and significant) correlation of $r=.422, p=.001$. Because there was a positive correlation, results suggested that if the higher the score was achieved on Ekman's Emotion Hexagon Test, then the higher the score would also be achieved on Laura's Emotion Hexagon Test.

Please see table 2 for the correlational analysis statistics.

Table 2. Correlations between all personality and emotion task variables.

\begin{tabular}{|l|l|l|l|l|l|l|l|}
\hline & & $\mathbf{2}$ & 3 & 4 & 5 & 6 \\
\hline 1 & Laura's Test & & & & & & \\
\hline 2 & Ekman's Test & $.422^{\star \star}$ & & & & & \\
\hline 3 & Openness & .114 & .248 & & & & \\
\hline 4 & Consciousness & .015 & .144 & .246 & & & \\
\hline 5 & Agreeableness & .226 & .147 & .243 & $.443^{\star \star}$ & & \\
\hline 6 & Extraversion & $-.268^{\star}$ & .028 & $.400^{\star \star}$ & $.341^{\star \star}$ & $.260^{\star}$ & \\
\hline 7 & Neuroticism & .105 & .119 & -.155 & -.195 & $-.399^{\star \star}$ & $-.393^{\star}$ \\
\hline
\end{tabular}

Table Notes: ${ }^{*} p .05,{ }^{* *} p<.01,{ }^{* * *} p<.001$

\section{Sensitivity of Overall Task to Personality Variables}

Two multiple regression analyses were then ran. The first was between Laura's Emotion Hexagon Task and all fivepersonality traits (openness, conscientiousness, agreeableness, extraversion and neuroticism). This analysis presented a significant model, $R^{2}=.459, F(5,54)=2.876, p=.023$. The two personality traits that were found to be significant predictors of Laura's Emotion Hexagon Task score were extraversion $t(54)=-2.695, p=.009$ and agreeableness $, t(54)=2.242, p=.029$. As extraversion has a negative Beta value of -.393 , this demonstrates the negative relationship between the score of Laura's Emotion Hexagon Task and extraversion. In contrast, agreeableness has a positive Beta value of .319, demonstrating the positive relationship between the score of Laura's Emotion Hexagon Task and agreeableness. Please see Table 3 for the regression statistics for this model.

The second regression analysis was between Ekman's Emotion Hexagon Test and all five-personality traits. In this case, the regression model was not significant suggesting that not one of the five personality traits could predict an individual's score on Ekman's Emotion Hexagon Test. Please see table 4 for the regression statistics for this model although it did not reach significance.

Table 3. Regression statistics the regression of the five personality predicting Laura's Emotion Hexagon Test

\begin{tabular}{|l|l|l|l|l|l|l|}
\hline & $\mathrm{B}$ & $\boldsymbol{T}$ & Beta & $\boldsymbol{R}$ & $\boldsymbol{R}^{\mathbf{2}}$ & $\Delta \mathrm{R}^{2}$ \\
\hline Model & & & & .459 & .210 & .137 \\
\hline Openness & .136 & 1.593 & .214 & & & \\
\hline Consciousness & -.014 & -.199 & -.028 & & & \\
\hline Agreeableness & .149 & $2.242^{\star}$ & .319 & & & \\
\hline Extraversion & -.188 & $-2.695^{\star \star}$ & -.393 & & & \\
\hline Neuroticism & .043 & .632 & .086 & & & \\
\hline
\end{tabular}

Table 4. Regression statistics the regression of the five personality predicting Ekman's Emotion Hexagon Test

\begin{tabular}{|l|l|l|l|l|l|l|}
\hline & $\mathbf{B}$ & $\boldsymbol{T}$ & Beta & $\boldsymbol{R}$ & $\boldsymbol{R}^{\mathbf{2}}$ & $\Delta \mathbf{R}^{\mathbf{2}}$ \\
\hline Model & & & & .339 & .115 & .033 \\
\hline Openness & .251 & 1.758 & .250 & & & \\
\hline Consciousness & .063 & .550 & .082 & & & \\
\hline Agreeableness & .098 & .877 & .132 & & & \\
\hline Extraversion & -.043 & -.371 & -.057 & & & \\
\hline Neuroticism & .155 & 1.353 & .196 & & & \\
\hline
\end{tabular}

\section{Openness Trait}

One of the aims of this investigation was to look in more detail at the personality trait of openness. Despite the regression analysis showing little detail about the openness trait, a t-test analysis provided further details.

Scores for the trait of openness were categorised into 'high' and 'low' scores. High scores were those over 52 and low scores were those under 48.

The t-test analysis demonstrated that openness does have a significant effect upon an individual's facial emotion recognition in Laura's Emotion Hexagon Test, $t(58)=-2.740$, $p=.008$. The people who were seen to have low openness had a mean task score of 79.8(11.46) in comparison to the high openness people who scored 92.57(11.95).

In contrast to this, openness does not have a significant effect on an individual's facial emotion recognition in Ekman's Emotion Hexagon Test, $t(58)=1.414, p=.133$. This result both supported and did not support the first prediction made by 
researchers. As the t-test only proved a significant effect of openness upon Laura's Emotion Hexagon Task, the researchers' prediction cannot be said to be truly correct and supported.

\section{Individual Emotions}

Regressions looked at whether the Big Five Personality Traits (openness, conscientiousness, extraversion, agreeableness and neuroticism) could predict the scores for the individual emotions within Laura's Emotion Hexagon Test. These emotions were happiness, sadness, disgust, fear, anger and surprise.

Regressions demonstrated that personality predicted an individual's performance on one of the six basic emotions within Laura's Emotion Hexagon Task. This emotion was sadness, $R^{2}=.239, F(5,54)=3.390, p=.010$.Significant predictors of disgust were both agreeableness, $t(58)=2.613, p=.012$, with a positive Beta value of .362 and extraversion, $t(58)=$ $-2.683, p=.010$, with a negative Beta value of -.384 .

Another set of regressions looked to see if the five personality traits (openness, conscientiousness, extraversion, agreeableness and neuroticism) could predict the scores for the individual emotions within Ekman's Emotion Hexagon Test. Again, regressions demonstrated that personality can be used to predict an individual's performance on one of the six basic emotions within Ekman's Emotion Hexagon Test. This emotion was disgust, $R^{2}=.498, F(5,54)=3.566, p=.007$. The only significant predictor of disgust was neuroticism, $t(58)=3.444$, $p=.001$, with a positive Beta value of .459 .

\section{Discussion}

The aim of this investigation was to see if personality could predict the scores on the two facial emotion recognition tasks. Because there had been previous issues regarding the different emotion recognition methods, a new method (Laura's Emotion Recognition Task) was created and compared an older method of Ekman's Emotion Hexagon Test.

Findings from this study demonstrated that both Laura's Emotion Hexagon Task and Ekman's Emotion Hexagon Test were appropriate measures of facial emotion recognition. Results showed that the personality traits of agreeableness and extraversion were predictors of facial emotion recognition concerning Laura's Emotion Hexagon Task. The analysis relating Ekman's Emotion Hexagon Test had no significant predictions. The result of a further analysis indicated that openness did have an effect on an individual's facial emotion recognition only when participants had completed Laura's Emotion Recognition Task. The participant who has low openness also had lower task scores in this case.

The current researchers made the prediction that openness could predict how well an individual would score on both Laura's Emotion Hexagon Task and Ekman's Emotion Hexagon Test. This prediction had no support. Researchers also predicted that the other four personality variables (extraversion, agreeableness, conscientiousness and neuroticism) would not have any significant effect on an individual's facial emotion recognition score on both tasks. This again had no support from the results. Finally, it was predicted that there would be some form of correlation between Laura's Emotion Hexagon Task and Ekman's Emotion Hexagon Test. This prediction was supported.

The findings from this investigation did not show full support for previous research [21] [18].

Terracciano, Merritt, Zonderman and Evans [21] suggested that facial emotion recognition was predicted by a person's personality trait of openness, and slightly by the trait of conscientiousness. They found no significant results with agreeableness, extraversion or neuroticism. This study, on the other hand, found that openness, agreeableness and extraversion is to predict an individual's facial emotion recognition when participants completed Laura's Emotion Hexagon Task.

Mill, Allik, Realo and Valk [18] found that both openness and conscientiousness correlated with their facial emotion recognition task. This is slightly different to the current study who found correlations with agreeableness, extraversion and a significant effect of openness when being concerned with participants' facial emotion recognition on Laura's Emotion Hexagon Task. There are many reasons why the results of both studies could have differed.

One possible difference could have been because there were different methods used in each study. Terracciano, Merritt, Zonderman and Evans [21] had used the Perception of Affect Task (PAT) which has a different structure to Ekman's Emotion Hexagon Test. Participants are not asked to look at morphed images in the PAT so therefore the methods used by Terracciano could be seen as an easier test to take.

Mill, Allik, Realo and Valk [18] had also used a different method when assessing facial emotion recognition. In this case, the Japanese and Caucasian Facial Expressions of Emotion (JACFEE) was used. Despite using images taken from 'Picutres of Facial Affect' (Ekman and Friesen), not all the photographs were placed in the same order and the photographs were not morphed giving the test a slightly lower level of difficulty. Mill, Allik, Realo and Valk [18] showed participants only 32 images with the use of the Japanese and Caucasian Facial Expressions of Emotion. This could have been one of the main reasons for the difference in results compared to the current research. Both Laura's Emotion Hexagon Task and Ekman's Emotion Hexagon Test gave participants the opportunity to look at 150 images of emotion expressions in the human face. Suggestions could propose that the larger amount of photographs allowed for participants to gain more experience in identifying the emotions in the images. This in turn would mean that participants' scores could be higher when recognising the six basic emotions.

One other possible reason for the difference in results could have been due to the samples in each study. The current study used 60 Northumbria University students whereas Terracciano et al. [21] use 152 participants (African American and Hispanic). There are many differences with these samples, which could mean that one sample is better than the other is at recognising 
emotions in the human face. Factors include things such as culture, education and socioeconomic status. Because there has been little research done in this area, these reasons can only be a possibility (and can only be suggested) because they do not have empirical support. We cannot be sure whether individuals from different cultures would perform the same on Laura's Emotion Hexagon Task, Ekman's Emotion Hexagon Test, The Japanese and Caucasian Facial Expressions of Emotion, and the Perception of Affect Task. Cross-cultural investigations could be an option to help discuss this issue.

Another possible reason (relating to the participants) could have been the differences for exposure individuals had previously had to each emotion recognition task. The current study used Psychology students who will have more than likely covered the topic of emotion recognition somewhere within the Psychology Degree, including the tasks used. Both Terracciano et al. [21] and Mill et al. [18] did not use students so therefore their exposure to both emotion recognition tasks could be more limited. Any differences in results could have been down to the fact of more exposure and more practice from the participants of the current study. In order to control this, researchers could have noted down whether participants had encountered Ekman's Emotion Hexagon Test prior to the testing phase.

When analysing results, a positive correlation between both Laura's Emotion Hexagon Task and Ekman's Emotion Hexagon Test was presented. This gives support to suggest that Laura's Emotion Hexagon Task is a very good measure of facial emotion recognition. If participants did well on Laura's Emotion Hexagon Task then they would also do well on Ekman's Emotion Hexagon Test.

From looking at the statistical averages calculated from the data, results demonstrated that participants had performed better on Ekman's Emotion Hexagon Test compared to Laura's Emotion Hexagon Task. There are again many possible reasons for this difference.

The first reason can relate to the reliability and validity of each facial emotion recognition test. Ekman's Emotion Hexagon Test has been shown to have very good split-half reliability (FEEST Manual) whereas Laura's Emotion Hexagon Task does has not have any previous research conducted using the task itself. In order to compare the two facial emotion recognition tasks further, investigations about the reliability of Laura's Emotion Hexagon Task needs to be considered.

Calder et al. [4] had given support for the validity of Ekman's Emotion Hexagon Test by using it to look at emotion recognition within the adult life span. Because the test was used successfully on a number of occasions, this gave researchers a good basis to say that Ekman's Emotion Hexagon Test was a valid method of assessing the recognition of emotion in the human face.

Because the current study is the first piece of research to use Laura's Emotion Hexagon Task, researchers cannot conclude that this method is valid. Even though Laura's Emotion Hexagon Task was created in the same way as Ekman's Emotion Hexagon Test was created (using the same number of photographs with similar ratios of emotions), it cannot be seen as valid until further research is conducted using the test in different situations.

The final reason for the difference in results could simply be down to the involvement of order effects. All participants completed Laura's Emotion Hexagon Task before they completed Ekman's Emotion Hexagon Test. Results could possibly have been different if participants completed the tasks in a different order, therefore reducing the involvement of order effects.

One of the main aims of this investigation was to look at the argument surrounding the photographs in Ekman's Emotion Hexagon Test (and the earlier methods). The argument put forward by Suzuki et al. [20] suggested that participants were finding it very easy to recognise the emotions within the photographs. This was simply because the photographs are becoming universal and are being displayed on many occasions. Suzuki et al. [20] suggested that recent methods are still using the photographs taken from 'Pictures of Facial Affect' (Ekman and Friesen) so therefore anyone with background knowledge of these images would find the task very easy.

Laura's Emotion Hexagon Task helps to provide evidence for this argument. Because participants performed better in Ekman's Emotion Hexagon Test (with a higher average than in Laura's Emotion Hexagon Task), it can be suggested that Suzuki et al. [20] were right in some ways.

The current study also looked to see if any individual basic emotion scores (happiness, sadness, fear, disgust, surprise and anger) could be predicted by an individual's personality. This was not one of the main aims but results gave some good points to discuss. Results (from the currentstudy) demonstrated that in Laura's Emotion Hexagon Task, only the detection of the sadness emotion could be predicted by the five personality traits. In the case of Ekman's Emotion Hexagon Test, the only emotion that could be predicted by the five personality traits was the emotion of disgust.

Very little research uses the 'normal population' so therefore results will be discussed in relating to individuals with personality disorders.

Domes, Czieschnek, Weidler, Berger, Fast and Herpertz [8] looked at individuals who have a diagnosis of Borderline Personality Disorder to see if there were any links between the disorder and facial emotion recognition. Researchers asked 25 females to look at a set of stimuli that involved pictures created by Ekman and Friesen. It was demonstrated that the individuals who had Borderline Personality Disorder had no deficits in recognising emotions, but were able to identify the emotions of anger better than the other five basic emotions. Participants with Borderline Personality Disorder found it difficult to identify the emotion of surprise and fear amongst the pictures. Researchers suggested that these basic emotions could have had some sort of sensitivity to the Borderline Personality Disorder, therefore creating a difficulty in recognising the emotions.

Results from Domes, Czieschnek, Weidler, Berger, Fast and Herpertz [8] differ to the results demonstrated by the 
present study. From the present study, researchers had indicated a sensitivity towards to emotion of sadness (relating to Laura's Emotion Hexagon Task) and disgust (in Ekman's Emotion Hexagon Test).

Differences may have occurred because of the 'normal population' used in the current study. Because there were no specific personality disorders present in the current sample of participants, it could mean that the sensitivity to different emotions is also different. This study therefore gives an insight (and a direction) into the possibility of conducting further research using the normal population instead of conducting research-using individuals with specific disorders.

There are some areas of improvement if this investigation was repeated. Firstly, using live models to assess an individual's facial emotion recognition. Instead of having still images, participants could be asked to look at a model who is displaying one of the six basic emotions of anger, sadness, happiness, fear, disgust or surprise. This would increase the ecological validity of the investigation as you could put each participant in a real-life scenario where human interactions can take place.

One other improvement could be with the design of the experiment itself. The current study used a repeated measures design but there are also other options for the choice of design. To eliminate any order effects, a matched pairs design could have been used. Participants would have to match participants based on their personality types and other demographic factors (like age). This would mean that participants would not have to do both Ekman's Emotion Hexagon Test and Laura's Emotion Hexagon Task so it could be more difficult to make a comparison. One notable criticism of this study is that participants seemed to become very tired after completing the first of the two emotion recognition tasks. Eliminating fatigue effects would be done by counterbalancing the areas of the investigation.

With the current study in mind, there could be several areas for future research. The main area of investigation is the reliability and validity of Laura's Emotion Hexagon Task. Just because the task has worked once does not mean that the same results will occur again so the only way to look at this is to put the test in different situations.

By using the previous research findings related age [4] and emotional intelligence (Austin) [2], researchers could discover whether or not Laura's Emotion Hexagon Task could be used in these two situations just like Ekman's Emotion Hexagon was.

One other possible area for future research could be to look at individuals diagnosed with specific disorders. Sprengelmeyer et al. [19] looked at instances when individuals can lose the ability to recognise emotions correctly. Research was conducted using individuals who had been diagnosed with Huntington's disease - a disorder that can affect muscle control and can cause the de-generation of the brain. Participants recalled different facial photographs. These photographs were of both familiar faces and unfamiliar faces, all displaying different emotions. Results showed that participants with Huntington's disease were impaired in discriminating the emotion of anger and fear. Participants, however, showed the ability to discriminate between photographs displaying the happiness and sadness emotions, suggesting that there could be some problems with only negative emotion recognition. If the task has good reliability and validity then it could be used in situations with the nonnormal population.

\section{Conclusion}

In conclusion, the current study has demonstrated that Laura's Emotion Hexagon Task is a more up-dated facial emotion recognition task by initially presenting good reliability for this test. The task was used to show that personality does have a relation to facial emotion recognition in humans. Despite this finding, the validity of the new measure was not tested; therefore further investigations are needed to look specifically at the validity of this task. This, however, does not stop the opportunity for future research into the creation of new methodologies for assessing facial emotion recognition alongside the different variables that can affect their result.

\section{Conflict of interest}

The authors confirm that there is no conflict of interest regarding this manuscript.

\section{References}

1. Allik J, Laidra K, Realo A, Pullmann H. Personality development from 12 to 18 years of age: Changes in mean levels and structure of traits. European Journal of Personality. 2004; 18: 445-462. doi: 10.1002/per.524

2. Austin EJ. An investigation of the relationship between trait emotional intelligence and emotional task performance. Personality and Individual Differences. 2004; 36: 1855-1864. doi: 10.1016/j.paid.2003.07.006

3. Cacioppo JT, Gardner WL. Emotion. Annual Review of Psychology. 1999; 50: 191-214. doi: 10.1146/annurev.psych.50.1.191

4. Calder AJ, Keane J, Manly T, Sprengelmeyer R, Scott S, et al. Facial Expression Recognition Across The Adult Life Span. Neuropsychologia. 2003; 41: 195-202. doi: 10.1016/S0028-3932(02)00149-5

5. Calder AJ, Young AW, Rowland D, Perrett DI, Hodges JR, et al. Facial Emotion Recognition after Bilateral Amygdala Damage: Differentially Severe Impairment of Fear. Cognitive Neuropsychology. 1996; 13: 699-745. doi: 10.1080/026432996381890

6. Darwin C. The Expression of the Emotions in Man and Animals. London: Penguin Books Ltd; 1872-2009.

7. Diehl-Schmid J, Pohl C, Ruprecht C, Wagenpfeil S, Foerstl H, et al. The Ekman 60 Faces Test As A Diagnostic Instrument In Frontotemporal Dementia. Archives of Clinical Neuropsychology. 2007; 22: 459-464. doi: 10.1016/j.acn.2007.01.024

8. Domes G, Czieschnek D, Weidler F, Berger C, Fast K, et al. Recognition of Facial Affect in Borderline Personality Disorder. Journal of Personality Disorders. 2008; 22(2): 135-147. doi: 10.1521/pedi.2008.22.2.135

9. Ekman P. Universal Facial Expressions of Emotion. California Mental Health Readers Digest. 1970; 8(4): 151-158.

10. Ekman P. Darwin, Deception, and Facial Expression. Annual New York Academy of Sciences. 2003; 1000: 205-221. doi: 10.1196/annals.1280.010

11. Ekman P, Friesen WV, Tomkins SS. Facial Affect Scoring Technique: A First Validity Study. 1971. 
12. George MS, Ketter TA, Parekh PL, Horwitz B, Herscovitch $P$, et al. Brain activity during transient sadness and happiness in healthy women. Annual Journal of Psychiatry. 1995; 152: 341-351. doi: 10.1176/ajp.152.3.341

13. Goldberg LR. The IPIP-NEO: International Personality Item Pool Representation of the NEO PI-R. 1999.

14. Goldberg LR, Johnson JA, Eber HW, Hogan R, Ashton MC, et al. The International Personality Item Pool and the Future of Public-Domain Personality Measures. Journal of Research in Personality. 2006; 40: 84-96. doi: 10.1016/j.jrp.2005.08.007

15. Goleman D. Emotional Intelligence. New York: Bantam Publishing Company. 1995.

16. Matsumoto D, Ekman P. The relationship among expressions, labels, and descriptions of contempt. Journal of Personality and Social Psychology. 2004; 87: 529-540.

17. Matsumoto D, Le Roux J, Wilson-Cohn C, Raroque J, Kooken K, et al. A New Test To Measure Emotion Recognition Ability: Matsumoto and Ekman's Japanese and Caucasian Brief Affect Recognition Test (JACBART). Journal of Nonverbal Behaviour. 2000; 24(3): 179-209.
18. Mill A, Allik J, Realo A, Valk R. Age-Related Differences In Emotion Recognition Ability: A Cross-Sectional Study. Emotion. 2009; 5: 619-630. doi: $10.1037 / \mathrm{a} 0016562$

19. Sprengelmeyer R, Young AW, Calder AJ, Karnat A, Lange H, et al. Loss of Disgust Perception of Faces and Emotions in Huntington's Disease. Brain. 1996; 119: 1647-1665.

20. Suzuki A, Hoshino T, Shigemasu K. Measuing Individual Differences in Sensitivities to Basic Emotions in Faces. Cognition. 2006; 99: 327-353. doi: 10.1016/j.cognition.2005.04.003

21. Terracciano A, Merritt M, Zoderman AB, Evans MK. Personality Traits and Sex Differences in Emotions Recognitions among African Americans and Caucasians. Ann N Y Acad Sci. 2003; 1000: 309-312.

22. Young A, Perrett $D$, Calder A, Sprengelmeyer R, Ekman P. Facial Expressions of Emotion - Stimuli and Tests (FEEST). London: Thames Valley Test; 2002. 